\title{
Complications of Posterior Vertebral Resection for Spinal Deformity
}

\author{
Sung-Soo Kim, Beom-Cheol Cho, Jin-Hyok Kim, Dong-Ju Lim, Ji-Yong Park, Beom-Jung Lee, Se-II Suk \\ Department of Orthopaedics, Seoul Spine Institute, Inje University Sanggye Paik Hospital, \\ Inje University College of Medicine, Seoul, Korea
}

\begin{abstract}
Study Design: Retrospective study.
Purpose: To evaluate the incidence and risk factors of complications following posterior vertebral resection (PVR) for spinal deformity.
\end{abstract}

Methods: A review of 233 patients treated with PVR at one institution over a nine-year period (1997 to 2005) was performed. The average age was 33.5 years. Complications were assessed in terms of surgical techniques (posterior vertebral column resection [PVCR] and decancellation osteotomy) and etiologies of deformity.

Results: Local kyphosis was corrected from $51.4^{\circ}$ to $2.7^{\circ}$, thoracic scoliosis $63.9^{\circ}$ to $24.5^{\circ}(62.6 \%$ correction), and thoracolumbar or lumbar scoliosis $50.1^{\circ}$ to $17.1^{\circ}$ (67.6\%). The overall incidence of complications was $40.3 \%$. There was no significant difference between PVCR and decancellation osteotomy in the incidence of complications. There were more complications in the older patients ( 35 years) than the younger $(p<0.05$ ). Higher than 3,000 $\mathrm{ml}$ of blood loss and $200 \mathrm{minutes}$ of operation time increased the incidence of complications, with significant difference $(p<0.05)$. More than 5 levels of fusion significantly increased the total number of complications and postoperative neurologic deficit $(p<0.05)$. Most of the postoperative paraplegia cases had preoperative neurologic deficit. Preoperative kyphosis, especially in tuberculous sequela, had higher incidences of complications and postoperative neurologic deficit $p<0.05$ ). More than $40^{\circ}$ of kyphosis correction had the tendency to increase complications and postoperative neurologic deficit without statistical significance $(p>0.05)$. There was 1 mortality case by heart failure. Revision surgery was performed in 15 patients for metal failure or progressing curve.

Conclusions: The overall incidence of complications of PVR was 40.3\%. Older age, abundant blood loss, preoperative kyphosis, and long fusion were risk factors for complications.

Key words: Posterior vertebral resection, Posterior vertebral column resection, Decancellation osteotomy, Postoperative complications

\section{Introduction}

Vertebral resection is a demanding and difficult surgical technique reserved for severe and/or rigid spinal deformities that cannot be brought to acceptable range of correction and balance with less aggressive methods [1-9].
There are two kinds of posterior vertebral resection which remove three columns of vertebra: decancellation osteotomy and posterior vertebral column resection (PVCR) $[4,10-$ 12]. Decancellation osteotomy is often expressed as eggshell procedure or pedicle subtraction osteotomy [12,13]. This resection, eliminating the posterior elements, pedicles,

Received Oct 11, 2012; Revised Oct 17, 2012; Accepted Oct 18, 2012

Corresponding author: Dong-Ju Lim, MD

Department of Orthopaedics, Seoul Spine Institute, Inje University Sanggye Paik Hospital, Inje University College of Medicine, 1342 Dongil-ro, Nowon-gu, Seoul 139-707, Korea

Tel: +82-2-950-1288, Fax:+82-2-950-6342, E-mail: scd25@paik.ac.kr 
and vertebral body through the posterior approach, makes a V-shaped space in the vertebra. The space is then closed bone-on-bone with anterior hinge. Since Heinig and Boyd [10] introduced the posterior egg-shell procedure for sagittal deformity, it has been widely performed in ankylosing spondylitis, posttraumatic kyphosis, and other severe deformities.

PVCR, eliminating the posterior elements, pedicles, and the entire vertebral body with the discs above and below, makes a trapezoid space in the spine column. Closure of the space usually creates an unsupported gap which requires an anterior reconstruction. PVCR is used to remove at least one vertebral segment. This technique can be performed either through the combined anterior and posterior approach or through a single posterior approach $[2,3]$. Since verterbrectomy was first illustrated by MacLennan [11], who described posterior apical resection, posterior vertebral resection (PVR) has been used in the treatment of congenital deformity, fixed coronal deformity, and other severe deformities. Bradford and Tribus $[14,15]$ reported his experience of the combined anterior and PVCR for fixed coronal decompensation, and the authors [2] in the previous report presented the results of PVCR for severe spinal deformity.

In vertebral resection, kyphosis can be corrected with bilateral symmetric resection, and scoliosis can be corrected with asymmetric resection in coronal plane [3].

Although several investigators have reported on the complications of decancellation osteotomy and PVCR $[1,13,15,16]$, there have been few reports comparing the complications of the two resection techniques and their suggested risk factors.

The present study was undertaken to evaluate the incidence and risk factors of complications following PVR for spinal deformity and compare the results of decancellation osteotomy and PVCR.

\section{Materials and Methods}

Two hundred thirty-three patients treated with PVR and pedicle screw instrumentation during the period of 1997 to 2006 were retrospectively analyzed. The mean age of patients at the time of surgery was 33.5 years (range, 2 to 76), and the minimum follow-up period was 2 years (range, 2 to 9.7). There were 121 females and 112 males.

The clinical records and radiographs of all patients were reviewed. The clinical records were reviewed for demographic data, etiology of spinal deformity, operation time, blood loss, and complications. To evaluate complications using clinical records, the records were categorized into transient neurologic deficit, permanent neurologic deficit, infection, and miscellaneous. Postoperative neurologic deficit was evaluated with transient neurologic deficit and permanent neurologic deficit. Transient neurologic deficit refers to aggravating neurologic deficit immediately after surgery as compared to the preoperative state and recovering from it during the follow-up. Permanent neurologic deficit refers to aggravating and persisting neurologic deficit at immediate postoperative and follow-up period as compared to the preoperative state, such as complete cord injury, complete cauda equina injury, and other irreversible neurologic deficit. Miscellaneous complications except for the neurologic deficit and infection included conditions that adversely affected the recovery of the patient, such as dural tear, hemothorax, pleural effusion, pulmonary embolism, and major abdominal ileus.

The radiographic materials using the standing anteroposterior and lateral spine scanogram were reviewed for deformity correction, complication related to instrumentation (instrument failure), and progressing curve. Deformity in coronal and sagittal plane was measured by the Cobb method. Instrument failure included the broken rod and progressive proximal or distal screw pullout during the followup. Progressing curve was defined as increasing deformity of more than $5^{\circ}$ in the fusion mass or adjacent to the fusion mass.

Complications were assessed in terms of surgical techniques (PVCR vs. decancellation osteotomy) and etiologies of deformity. To analyze risk factors of complications, the effects of patient age, gender, etiology, resection level, the number of segments resected or fused, usage of titanium mesh, operation time, and blood loss on the development of complications were evaluated.

Statistical analysis was performed using MedCalc for Windows, ver. 12.3. (MedCalc Software, Mariakerke, Belgium). The data was analyzed using the Student's $t$-test, Pearson chi-square test, and Fisher's exact test. A $p$-value of less than 0.05 was considered statistically significant.

\section{Surgical techniques}

All surgeries were carried out under the somatosensory evoked potential monitoring.

With the spine exposed posteriorly, pedicle screws were inserted segmentally, except for the resected levels, using 
intraoperative posteroanterior and lateral X-rays for localization of pedicles. The screws were connected on one side with a temporary rod contoured to the shape of the deformity. After a wide laminectomy and bilateral foraminotomies to decompress the neural elements, vertebral resection at the apex of the deformity was carried out.

In the thoracic spine, the rib heads were removed to obtain access to the lateral wall of the vertebral body and to allow untethered motion of the vertebral column. In lumbar spine, the transverse processes were osteotomoized at base with soft tissue around the left. Meticulous subperiosteal dissection was performed down to the lateral wall of the vertebral body until the anterior surface of the body was easily palpable. Segmental vessels were carefully preserved. If segmental vessels were injured during dissection, the bleeding was controlled by electric cauterization and gelfoam soaked with thrombine. Under direct visualization, the pedicles and the lateral portions of the vertebral body were removed using a small osteotome. Then, the vertebra was resected piecemeal from lateral to medial through the space created by the resection, but not resected past the midline. A thin rim of posterior vertebral wall beneath the dura was left intact, to be removed at a later time. The anterior wall was also removed in the piecemeal fashion, taking care to leave intact the soft tissue tube anterior to the vertebral body. When an adequate amount of vertebral body was removed, the entire portion of the posterior vertebral wall over to the midline was removed with an Epstein reverse cutting curette and pituitary forceps. Following the resection of the posterior wall on the working side, another temporary rod was inserted to the working side and securely locked to the screws with slight compression to shorten the vertebral column. Then the temporary rod on the opposite side was removed to allow resection of the remaining vertebra. The same sequence of resection was carried out on the remaining vertebra. In decancellation osteotomy, V-shape space was made by resecting both the pedicles and adjacent vertebral body. In PVCR, trapezoid space was made by resecting at least one vertebral body with the discs above and below. For the correction of kyphosis, symmetric resection was performed on both sides, and for the correction of scoliosis, asymmetric resection was performed with more resection of the convex side. At the completion of the resection, the rod that had been removed was replaced and secured with the screws. Then, a final evaluation of the spinal canal was performed to confirm that there was no residual compression at the resected margins, and that no bony or disc tissue anterior
Table 1. Dermographics and etiologic diagnosis

\begin{tabular}{lc}
\hline \hline Factors & \\
\hline Age (yr) & $33.5(2-76)$ \\
Female/male & $121 / 112$ \\
Follow-up (yr) & $6.1(2-9.6)$ \\
Diagnosis (pts.) & \\
Congenital deformity & 103 \\
Posttuberculous kyphosis & 39 \\
Posttraumatic kyphosis & 26 \\
Ankylosing spondylitis & 22 \\
Adult deformity & 22 \\
Neuromuscular scoliosis & 8 \\
Others & 13 \\
Deformity (pts.) & \\
Scoliosis & 117 \\
Kyphosis & 178 \\
\hline
\end{tabular}

Pts: Patients.

to dura were left to hinder free, untethered movement of the dural tube.

Deformity correction was carried out by replacing the temporary rods with rods pre-contoured to the corrected shape. To avoid inadvertent distraction of the neural elements, the vertebral column was initially shortened by slight compression over the resected gap without locking of the temporary rods. The deformity was gradually corrected with repeated additional compression to shorten the vertebral column. The compression and shortening over the resected gap was carried out until the exposed dura looked redundant. After compression and shortening of the resected gap, the temporary rod was changed to pre-contoured final rods one by one to avoid any loss of shortening of the resected gap.

In decancellation osteotomy, V-shape space formed by resection was usually closed bone-on-bone without requiring anterior bone graft. Sometimes the small gap after closure was packed with autogenous cancellous chip bone graft. In PVCR, trapezoid space usually created unsupported gap requiring anterior bone graft after closure. Autogenous cancellous chip bone graft with/without titanium mesh was used for unsupported anterior gap. If the anterior interbody gap was less than $5 \mathrm{~mm}$, autogenous cancellous chip bone was inserted. If the gap was longer than $5 \mathrm{~mm}$, a titanium mesh cage filled with bone chips was inserted, and additional autogenous iliac bone was put around the titanium mesh. After placement of the bone graft, the wound was closed over 
Table 2. Complications according to surgical techniques and diagnosis $(n=233)$

\begin{tabular}{|c|c|c|c|c|c|c|c|}
\hline & $\begin{array}{c}\text { Transient } \\
\text { N }\end{array}$ & $\begin{array}{l}\text { Permanent } \\
\mathrm{N}\end{array}$ & IF or PC & Wound Infection & Dura tear & Miscellaneous & Incidence \\
\hline \multicolumn{8}{|l|}{ Surgical techniques } \\
\hline PVCR (152) & $21(13.8)$ & $5(3.3)$ & $12(7.9)$ & $10(6.6)$ & $16(10.5)$ & $6(3.9)$ & $60(39.5)$ \\
\hline DO (81) & $6(7.4)$ & $1(1.2)$ & $13(16.0)$ & $4(4.9)$ & $7(8.6)$ & $5(6.2)$ & $34(42.0)$ \\
\hline \multicolumn{8}{|l|}{ Diagnosis } \\
\hline Congenital D (103) & $9(8.7)$ & $2(1.9)$ & $8(7.8)$ & $6(5.8)$ & $10(9.7)$ & $5(4.9)$ & $34(33.0)$ \\
\hline Posttuberculous (39) & $10(25.6)$ & $3(7.7)$ & $4(10.3)$ & $3(7.7)$ & $4(10.3)$ & 0 & $21(53.8)$ \\
\hline PTK (26) & $5(19.2)$ & $1(3.8)$ & $3(11.5)$ & $1(3.8)$ & $5(17.2)$ & $1(3.8)$ & $13(50.0)$ \\
\hline AS (22) & $1(4.5)$ & $0(0)$ & $3(13.6)$ & $0(0)$ & $1(4.5)$ & $1(4.5)$ & $6(27.3)$ \\
\hline Adult deformity (22) & $1(4.5)$ & $0(0)$ & $6(27.3)$ & $1(4.5)$ & 0 & $1(4.5)$ & 9 (40.9) \\
\hline Others $(21)$ & $1(4.8)$ & $0(0)$ & $1(4.8)$ & $3(14.3)$ & $3(14.3)$ & $3(14.3)$ & $11(52.3)$ \\
\hline Total & $27(11.6)$ & $6(2.6)$ & $25(10.7)$ & $14(6.0)$ & 23 & $11(4.7)$ & $94(40.3)$ \\
\hline
\end{tabular}

Values are presented as number $(\%)$.

Transient N: Transient neurologic deficit, Permanent N: Permanent neurologic deficit, IF: Instrument failure, PC: Progressing curve, PVCR; Posterior vertebral column resection, DO: Decancellation osteotomy, Congenital D: Congenital deformity, Posttuberculous: Posttuberculous kyphosis, PTK: Posttraumatic kyphosis, AS: Ankylosing spondylitis.

suction drains.

\section{Results}

Etiologic diagnoses were congenital deformity in 103 patients, posttuberculous kyphosis in 39, posttraumatic kyphosis in 26 , ankylosing spondylitis in 22, adult deformity in 22, and miscellaneous in 21 . One hundred seventeen patients had scoliosis with coronal curve of greater than $20^{\circ}$, and 178 patients had kyphosis with sagittal curve of greater than $0^{\circ}$. Sixty-three patients had kyphoscoliosis with coronal curve $>20^{\circ}$ and sagittal curve $>0^{\circ}$ (Table 1).

The mean preoperative major coronal curve of scoliosis patients was $59.2^{\circ} \pm 25.7^{\circ}$, and the mean preoperative sagittal curve of kyphosis patients was $51.4^{\circ} \pm 30.7^{\circ}$.

\section{The results of operative procedures}

The average number of vertebrae resected was $1.5 \pm 1.2$ with fusion extent of $5.8 \pm 3.0$ segments. The resection was performed at the thoracic level in 95 patients and at lumbar level in 138 patients. The average operation time was $268 \pm$ 82 minutes, with the estimated blood loss of 4,392 $\pm 3,576$ $\mathrm{ml}$.

Eighty-one patients had undergone decancellation osteotomy and 152 patients had undergone PVCR. Among patients using PVCR, titanium mesh was inserted for anterior column reconstruction in 66.

In patients with scoliosis $(\mathrm{n}=117)$, the mean preoperative major coronal curve of $59.2^{\circ} \pm 25.7^{\circ}$ was corrected to $19.6^{\circ} \pm 15.0^{\circ}$ immediately after surgery, with a correction of $68.6 \%$. At the final follow-up, the coronal curve was $21.6^{\circ}$ $\pm 16.2^{\circ}$ with a correction of $64.6 \%$, demonstrating a correction loss of 3.4\% during the follow-up period. In patients with kyphosis $(\mathrm{n}=178)$, the mean preoperative major sagittal curve of $51.4^{\circ} \pm 30.7^{\circ}$ was corrected to $2.7^{\circ} \pm 23.8^{\circ}\left(48.7^{\circ}\right.$ correction) immediately after surgery and $8.0^{\circ} \pm 24.0^{\circ}\left(43.8^{\circ}\right.$ correction) at the final follow-up, demonstrating a correction loss of $4.9^{\circ}$ during the follow-up period.

At the final follow-up, the average correction rate of scoliosis was $50.9 \%$ in 17 patients using decancellation osteotomy and $66.8 \%$ in 100 patients using PVCR, with statistically significant difference $(p=0.0028)$. The average correction degree of kyphosis was $40.0^{\circ}$ in 76 patients using decancellation osteotomy and $51.5^{\circ}$ in 102 patients using PVCR, with statistically significant difference $(p=0.011)$.

\section{The results of complications}

Complications were classified into transient neurologic deficit, permanent neurologic deficit, instrument failure and/ or progressing curve, wound infection, and miscellaneous. The details of complications according to surgical techniques (decancellation osteotomy vs. PVCR) and etiologic 
Table 3. Associated factors and their significances of complications

\begin{tabular}{|c|c|c|c|c|c|}
\hline \multirow{2}{*}{ Factors } & \multirow{2}{*}{ Grouping } & \multicolumn{2}{|c|}{ Complication } & \multirow{2}{*}{$\begin{array}{c}\text { Odds ratio } \\
\text { (CI) }\end{array}$} & \multirow{2}{*}{$p$-value } \\
\hline & & Yes & No & & \\
\hline \multirow[t]{2}{*}{ Gender } & Female & 54 & 67 & 1.16 & 0.678 \\
\hline & Male & 46 & 66 & $(0.69-1.94)$ & \\
\hline \multirow[t]{2}{*}{ Age (yr) } & $>35$ & 55 & 50 & 2.03 & 0.012 \\
\hline & $\leq 35$ & 45 & 83 & $(1.20-3.44)$ & \\
\hline \multirow[t]{2}{*}{ Diagnosis } & Posttuberculous & 23 & 16 & 2.18 & 0.041 \\
\hline & Others & 77 & 117 & $(1.08-4.40)$ & \\
\hline \multirow[t]{2}{*}{ Kyphosis } & Yes & 89 & 89 & 3.91 & 0.0002 \\
\hline & No & 11 & 43 & $(1.89-8.07)$ & \\
\hline \multirow[t]{2}{*}{ Scoliosis } & Yes & 45 & 72 & 0.70 & 0.273 \\
\hline & No & 54 & 61 & $(0.42-1.19)$ & \\
\hline \multirow[t]{2}{*}{ Preop. neurologic deficit } & Yes & 25 & 8 & 5.21 & 0.0001 \\
\hline & No & 75 & 125 & $(2.23-12.14)$ & \\
\hline \multirow[t]{2}{*}{ Surgical technique } & Decancellation osteotomy & 37 & 44 & 1.19 & 0.629 \\
\hline & PVCR & 63 & 89 & $(0.69-2.05)$ & \\
\hline \multirow[t]{2}{*}{ Resection level } & Thoracic spine & 49 & 46 & 1.82 & 0.037 \\
\hline & Lumbar spine & 51 & 87 & $(1.07-3.09)$ & \\
\hline \multirow[t]{2}{*}{ No. of fusion extent (segments) } & $>6$ & 57 & 46 & 2.51 & 0.001 \\
\hline & $\leq 5$ & 43 & 87 & $(1.47-4.27)$ & \\
\hline \multirow{2}{*}{$\begin{array}{l}\text { No. of vertebrae resected } \\
\text { (segments) }\end{array}$} & $\geq 2$ & 29 & 19 & 2.45 & 0.010 \\
\hline & 1 & 71 & 114 & $(1.28-4.69)$ & \\
\hline \multirow[t]{2}{*}{ Usage of mesh } & Yes & 35 & 31 & 1.77 & 0.070 \\
\hline & No & 65 & 102 & $(0.997-3.15)$ & \\
\hline \multirow[t]{2}{*}{ Operation time (min) } & $\geq 200$ & 80 & 85 & 2.26 & 0.012 \\
\hline & $<200$ & 20 & 48 & $(1.23-4.13)$ & \\
\hline \multirow[t]{2}{*}{ Blood loss (ml) } & $\geq 3,000$ & 61 & 52 & 2.44 & 0.001 \\
\hline & $<3,000$ & 39 & 81 & $(1.43-4.15)$ & \\
\hline \multirow[t]{2}{*}{ Kyphosis correction $\left(^{\circ}\right)$} & $\geq 40$ & 59 & 51 & 1.47 & 0.28 \\
\hline & $<40$ & 30 & 38 & $(0.80-2.69)$ & \\
\hline
\end{tabular}

diagnosis were shown in Table 2. There were 106 complications in 94 patients. The overall incidence of complications of PVR was 40.3\% (94/233). The postoperative neurologic deficit including transient and permanent neurologic deficit was found in 33 patients $(33 / 233,14 \%)$. Of the 33 patients, permanent neurologic deficit was detected in $6(6 / 233$, $2.6 \%$ ). Of 27 patients with transient neurologic deficit, 15 patients had hematoma, which were evacuated immediately. Seven patients had single nerve root injury at the lumbar level. Three patients had insufficient decompression around resection site. Two of the patients had no known reason for the transient neurologic deficit.

There were $25(25 / 233,10.7 \%)$ instrument failures and/ or progressing curves, which consisted of broken rods with progressing curves in 8 patients $(8 / 233,3.4 \%)$, proximal problems (proximal screws back-out or proximal adjacent kyphosis) in 7 (7/233, 3.0\%), distal problems (distal screws back-out or distal adjacent kyphosis) in 7 (7/233, 3.0\%), and distal adding-on deformity in 3 (3/233, 1.3\%). Revision surgery was performed in 15 patients $(15 / 233,6.4 \%)$. There 
Table 4. Risk factors of postoperative neurologic deficit

\begin{tabular}{|c|c|c|c|c|c|}
\hline \multirow{2}{*}{ Factors } & \multirow{2}{*}{ Grouping } & \multicolumn{2}{|c|}{ Complication } & \multirow{2}{*}{$\begin{array}{c}\text { Odds ratio } \\
(\mathrm{CI})\end{array}$} & \multirow{2}{*}{$p$-value } \\
\hline & & Yes & No & & \\
\hline \multirow[t]{2}{*}{ Diagnosis } & Posttuberculous & 15 & 24 & 4.23 & 0.0003 \\
\hline & Others & 26 & 169 & $(1.96-9.12)$ & \\
\hline \multirow[t]{2}{*}{ Kyphosis } & Yes & 37 & 141 & 4.46 & 0.007 \\
\hline & No & 3 & 51 & $(1.31-15.10)$ & \\
\hline \multirow[t]{2}{*}{ Preop. neurologic deficit } & Yes & 28 & 172 & 5.55 & 0.004 \\
\hline & No & 12 & 21 & $(2.47-12.47)$ & \\
\hline \multirow[t]{2}{*}{ No. of fusion extent (segments) } & $>6$ & 27 & 76 & 3.20 & 0.002 \\
\hline & $\leq 5$ & 13 & 117 & $(1.55-6.58)$ & \\
\hline \multirow[t]{2}{*}{ No. of vertebrae resected (segments) } & $\geq 2$ & 19 & 29 & 5.17 & $<0.0001$ \\
\hline & 1 & 21 & 164 & $(2.45-10.68)$ & \\
\hline \multirow[t]{2}{*}{ Insertion of mesh } & Yes & 21 & 45 & 3.64 & 0.0004 \\
\hline & No & 19 & 148 & $(1.80-7.35)$ & \\
\hline \multirow[t]{2}{*}{ Operation time (min) } & $\geq 200$ & 36 & 129 & 4.47 & 0.004 \\
\hline & $<200$ & 4 & 64 & $(1.52-13.09)$ & \\
\hline \multirow[t]{2}{*}{ Blood loss (ml) } & $\geq 3,000$ & 30 & 83 & 3.98 & 0.0004 \\
\hline & $<3,000$ & 10 & 110 & $(1.84-8.59)$ & \\
\hline
\end{tabular}

were 12 early infections and 2 late infections. All were treated by debridement and drainage. Instruments of 6 patients with 4 early infections and 2 later infections were removed due to recurrent infections.

There were 34 miscellaneous complications, which consisted of 23 dural tears, 6 hemothoraxes, 2 pleural effusions, 1 major abdominal ileus, 1 iliac donor site infection, and 1 mortality case. Most of the dural tears were found intraoperatively, but 3 dural tears were detected postoperatively with color and amount of drainage from the resection site. All dural tears detected during the operation were repaired directly or with graft if possible. Eight patients with hemothorax or plural effusion were managed by chest tube insertion. The patient with major abdominal ileus needed prolonged parenteral nutrition.

One patient with congenital kyphosoliosis died of heart failure without any definite pathology, intraoperatively.

\section{The risk factors of complications}

There was no significant difference in the incidence of complications between female and male $(p>0.05)$. Complications occurred more frequently in patients older than 35 years with significant difference $(p<0.05)$. The patients with the preoperative kyphosis had higher incidence of complications with significant difference, compared to those without kyphosis $(p<0.05)$. The patients with preoperative scoliosis, however, had a similar incidence rate compared to those without scoliosis $(p>0.05)$. Posttuberculous kyphosis increased complications with significant difference $(p<$ $0.05)$.

The complication rate was $42.0 \%$ in decancellation osteotomy and $39.5 \%$ in PVCR, without significant difference $(p>0.05)$. The preoperative neurologic deficit significantly increased complications $(p<0.05)$. The patients treated by resection at thoracic level had significantly higher incidence of complications than lumbar level $(p<0.05)$. The fusion extent of more than 5 segments and resection of 2 or more vertebrae increased complications with significant differences $(p<0.05)$. Insertion of titanium mesh for anterior column reconstruction had the tendency to increase complications, without significant difference $(p=0.070)$. The operation time of more than 200 minutes and blood loss of 3,000 ml were found to increase complications significantly $(p<0.05)$. Kyphosis correction of more than $40^{\circ}$ immediately after surgery was not found to increase complications $(p>0.05)$ (Table 3).

Multiple logistic regression analysis showed that the de- 
velopment of complications was significantly influenced by the preoperative neurologic deficit $(p=0.0035$, odds ratio $=$ $3.64)$, preoperative kyphosis $(p=0.0038$, odds ratio $=3.01)$, and fusion extent of more than 5 segments $(p=0.017$, odds ratio $=1.99$ ).

In the sub-analysis, the risk factors of postoperative neurologic deficit including transient and permanent neurologic deficit were evaluated. The preoperative kyphosis, posttuberculous kyphosis, preoperative neurologic deficit, fusion extent of more than 5 segments, resection of 2 or more vertebrae, insertion of titanium mesh, operation time of more than 200 minutes, and blood loss of more than 3,000 $\mathrm{ml}$ were risk factors to increase postoperative neurologic deficit and transient neurologic deficit with significant differences $(p<0.05)$ (Table 4). The patients older than 35 years and having the correction of kyphosis $\geq 40^{\circ}$ tended to have higher incidence of postoperative neurologic deficit $(p$ $=0.056)$ without significant differences $(p=0.056,0.0781)$. With multiple logistic regression analysis, it was found that the development of postoperative neurologic deficit was significantly influenced by resection of 2 or more vertebrae ( $p$ $=0.0001$, odds ratio $=4.73)$ and the preoperative neurologic deficit $(p=0.0011$, odds ratio $=3.04)$.

\section{Discussion}

Spinal deformity is a 3-dimensional deformity. Decompensation both in the coronal and the sagittal plane leads to specific clinical complaints, pain, neurologic deficit, progression of deformity, trunk imbalance, cardiopulmonary compromise, or interference with activities of daily living. In severe or rigid spinal deformities, conventional correction methods, such as posterior instrumentation or combined anterior release and posterior instrumentation, are usually unsatisfactory.

Vertebral resection removing three columns of vertebra makes a space, which is closed to correct deformity. Vertebral resection has been used for severe and/or rigid deformities with various etiologies, showing better deformity correction and balance than conventional correction methods. It is a technically demanding and exhausting procedure with high complication rates.

There are two kinds of vertebral resection, decancellation osteotomy (egg-shell procedure, pedicle subtraction osteotomy) and VCR.

Several authors have reported overall complications rate of decancellation osteotomy ranging from $15 \%$ to $60 \%$.
Murrey et al. [12] reported on 59 patients who underwent decancellation osteotomy. Ten patients had complications, such as pulmonary complication, instrument failure and infection, for a complication rate of $16.9 \%$. They had no postoperative neurologic complication. Willems et al. [17] presented complications of lumbar decancellation osteotomy for 62 patients with ankylosing spondylitis. Complications included $14.5 \%$ infections, $9.6 \%$ permanent neurologic deficit, $16 \%$ instrument failures, and $13 \%$ major general complications. The analysis of these data calculated the overall complication rate to be $53 \%$. Boachie-Adjei et al. [13] demonstrated the results of lumbar decancellation osteotomy for 24 patients with fixed sagittal deformity. They found 17 complications in 14 patients (58\%), which included 4 dural tears, 1 nerve root injury, 5 instrument-related problems, 1 hematoma, 1 infection, and others. Bridwell [16] reported an $8 \%$ incidence of neurologic deficit with decancellation.

In this series, the complication rate of decancellation osteotomy was $42 \%$. There were transient neurologic deficit in 6 patients $(7.4 \%)$, permanent neurologic deficit in $1(1.2 \%)$, instrument failure or progressing curve in $13(16 \%)$, wound infection in $4(4.9 \%)$, dura tear in $6(7.4 \%)$, and others in 2 (2.5\%). Our results were comparable to other studies.

There were few papers reporting on the complications of VCR. Bradford and Tribus [15] reported on 24 patients with rigid coronal decompensation who underwent combined anterior and posterior VCR. They had 31 complicatios in 14 patients (58\%). The most common complication was dural tear, which occurred in 8 patients. Other complications included wound infection in 3 patients, neurologic complication in 3 , and pseudarthrosis in 3 . In the previous report, the authors of this paper presented the results of PVCR for 70 spinal deformity patients. Twenty-four patients (34\%) had complications, which consisted of 2 complete cord injuries, 6 hematomas, 4 root injuries, 5 instrument failures, 2 infections, and 5 hemopneumothoraxes [2].

In this study, the complication rate of PVCR was $39.5 \%$. There were transient neurologic deficit in 21 patients (13.8\%), permanent neurologic deficit in $5(3.3 \%)$, instrument failure or progressing curve in $12(7.9 \%)$, wound infection in $10(6.6 \%)$, dura tear in $17(11.2 \%)$, and others in $9(5.9 \%)$.

Several authors categorized complications into major and minor in adult spinal deformity surgery [18-21]. In those studies, major complication usually included nerve injury, neurologic deficit, deep infection, fatal cardiopulmonary problem, and instrumentation failure. Minor complication 
included CSF leakage, superficial infection, and minor cardiopulmonary problem. Auerbach et al. [20] reported that major complications occurred in $35 \%$ of all 3 -column osteotomies, including $38 \%$ of pedicle subtraction osteotomy (decancellation osteotomy) and $22 \%$ of Vertebral column resection. Daubs et al. [18] reported the overall complication rate of $37 \%$, with the major complication rate of $20 \%$ in patients over 60 years of age, who underwent major spinal deformity surgery requiring a minimum 5-level arthrodesis procedure. In this study, the major complication rate of PVCR was $32 \%$ (49/152); the minor complication rate of PVCR was 14\% (21/152); and those of decancellation and osteotomy were $30 \%(24 / 81)$ and $15 \%(12 / 81)$, respectively.

For the first time, this study made a comparison of complications between decancellation osteotomy and PVCR and analyzed the data to find risk factors of complication for PVR, using many cases $(n=233)$. There was no significant difference in incidence of complication between decancellation osteotomy and PVCR.

Eight Risk factors of overall complications and postoperative neurologic deficit were found in this study. The most important risk factor of overall complication was the preoperative neurologic deficit, and preoperative kyphosis, followed by the fusion extent of more than 5 segments. Patients with preoperative neurologic deficit and kyphosis had 22 times higher complication rate compared to patients without both factors in the PVR surgery. Two important risk factors of postoperative neurologic deficit were the preoperative neurologic deficit and resection of 2 or more vertebrae. Patients with two risk factors had 29 times higher neurologic complication rate compared to patients without these factors in the PVR operation.

The authors confirm that the results obtained from this study will provide important information to patients and families with the indication of PVR operation. Furthermore, the identification of the risk factors of complications may play a major role in reducing the incidence of complications in PVR.

\section{Conclusions}

The overall incidence of complications of PVR was $40.3 \%$. The preoperative neurologic deficit, preoperative kyphosis, fusion extent of more than 5 segments, resection of 2 or more vertebrae, blood loss of more than $300 \mathrm{ml}$, operation time of more than 200 minutes, and age $>35$ were risk factors of complications of PVR.
Patients with the preoperative neurologic deficit and preoperative kyphosis had 22 times higher complication rate compared to patients without these factors in the PVR surgery. The patients with preoperative neurologic deficit and resection of 2 or more vertebrae increased 29 times in neurologic deficit in the comparison of patients with both factors.

\section{Acknowledgements}

This work was supported by Inje Research and Scholarship Foundation in 2010.

\section{REFERENCES}

1. Boachie-Adjei O, Bradford DS. Vertebral column resection and arthrodesis for complex spinal deformities. J Spinal Disord 1991;4:193-202.

2. Suk SI, Kim JH, Kim WJ, Lee SM, Chung ER, Nah KH. Posterior vertebral column resection for severe spinal deformities. Spine (Phila Pa 1976) 2002;27:237482.

3. Suk SI, Chung ER, Kim JH, Kim SS, Lee JS, Choi WK. Posterior vertebral column resection for severe rigid scoliosis. Spine (Phila Pa 1976) 2005;30:1682-7.

4. Smith-Petersen MN, Larson CB, Aufranc OE. Osteotomy of the spine for correction of flexion deformity in rheumatoid arthritis. Clin Orthop Relat Res 1969;66:69.

5. Leatherman KD, Dickson RA. Two-stage corrective surgery for congenital deformities of the spine. J Bone Joint Surg Br 1979;61:324-8.

6. Thomasen E. Vertebral osteotomy for correction of kyphosis in ankylosing spondylitis. Clin Orthop Relat Res 1985;(194):142-52.

7. Bradford DS. Vertebral column resection. Orthop Trans 1987;11:502.

8. Tokunaga M, Minami S, Kitahara H, Isobe K, Nakata Y, Moriya H. Vertebral decancellation for severe scoliosis. Spine (Phila Pa 1976) 2000;25:469-74.

9. Deviren V, Berven S, Smith JA, Emami A, Hu SS, Bradford DS. Excision of hemivertebrae in the management of congenital scoliosis involving the thoracic and thoracolumbar spine. J Bone Joint Surg Br 2001;83:496-500.

10. Heinig C, Boyd B. One stage vertebrectomy or eggshell procedure. Orthop Trans 1985;9:130. 
11. MacLennan A. Scoliosis. Br Med J 1922;2:864-6.

12. Murrey DB, Brigham CD, Kiebzak GM, Finger F, Chewning SJ. Transpedicular decompression and pedicle subtraction osteotomy (eggshell procedure): a retrospective review of 59 patients. Spine (Phila $\mathrm{Pa} 1976$ ) 2002;27:2338-45.

13. Boachie-Adjei O, Ferguson JA, Pigeon RG, Peskin MR. Transpedicular lumbar wedge resection osteotomy for fixed sagittal imbalance: surgical technique and early results. Spine (Phila Pa 1976) 2006;31:485-92.

14. Bradford DS, Tribus CB. Current concepts and management of patients with fixed decompensated spinal deformity. Clin Orthop Relat Res 1994;(306):64-72.

15. Bradford DS, Tribus CB. Vertebral column resection for the treatment of rigid coronal decompensation. Spine (Phila Pa 1976) 1997;22:1590-9.

16. Bridwell KH. Decision making regarding SmithPetersen vs. pedicle subtraction osteotomy vs. vertebral column resection for spinal deformity. Spine (Phila Pa 1976) 2006;31:S171-8.
17. Willems KF, Slot GH, Anderson PG, Pavlov PW, de Kleuver M. Spinal osteotomy in patients with ankylosing spondylitis: complications during first postoperative year. Spine (Phila Pa 1976) 2005;30:101-7.

18. Daubs MD, Lenke LG, Cheh G, Stobbs G, Bridwell KH. Adult spinal deformity surgery: complications and outcomes in patients over age 60. Spine (Phila Pa 1976) 2007;32:2238-44.

19. Glassman SD, Hamill CL, Bridwell KH, Schwab FJ, Dimar JR, Lowe TG. The impact of perioperative complications on clinical outcome in adult deformity surgery. Spine (Phila Pa 1976) 2007;32:2764-70.

20. Auerbach JD, Lenke LG, Bridwell KH, et al. Major complications and comparison between 3-column osteotomy techniques in 105 consecutive spinal deformity procedures. Spine (Phila Pa 1976) 2012;37:1198-210.

21. Schwab FJ, Hawkinson N, Lafage V, et al. Risk factors for major peri-operative complications in adult spinal deformity surgery: a multi-center review of 953 consecutive patients. Eur Spine J 2012;21:2603-10. 